\title{
Planets in Binary Stars
}

\section{E. Pilat-Lohinger ${ }^{\star a}$, Zs. Sándor $^{b}$, M. Gyergyovits ${ }^{a}$ and Á. Bazsó ${ }^{a}$}

${ }^{a}$ Institute of Astrophysics, University of Vienna, Türkenschanzstrasse 17, 1180 Vienna, Austria

${ }^{b}$ Eötvös Loránd University Budapest, Hungary

E-mail: elke.pilat-lohinger@univie.ac.at, szolt@caesar.elte.hu, markus.gyergyovits@univie.ac.at,akos.bazso@univie.ac.at

Discoveries of planets in binary star systems showed that planetary companions are not restricted to single stars and led to a growing interest in understanding planetary formation processes in general. In binary star systems, the proto-planetary disk is truncated due to the secondary star which has strong influence on the formation of planets. Despite numerous scientific studies thereto, we are still faced with many open questions regarding where and how a planet can grow in such environments as there are many parameters that affect the outcome of hydrodynamical simulations. In contrast thereto, terrestrial planet formation using gravitational N-body simulations can be easily performed. In either case, the architecture of a binary star- giant planet system influences the formation and evolution of additional planets. Furthermore, resonances play an important role for accretion processes and for the final set up of a planetary system.

Accretion Processes in Cosmic Sources - II - APCS2018

3-8 September 2018

Saint Petersburg, Russian Federation

\footnotetext{
* Speaker.
} 


\section{Introduction}

Observations suggest that a considerable fraction of stars in the solar neighbourhood are members of binary and multiple star systems. [1] and [2] established that in the solar neighborhood $(d<25$ parsec), about $40-45 \%$ of all Sun-like stars (spectral types F6-K3) are members of binary and multiple star systems, independent of whether or not they are hosting planets. Recently, [3] found for a sample of about 4800 F-/G-type main-sequence stars within 67 parsec of the Sun that $33 \%$ of the targets belong to binary star systems. The Washington Double Star Catalogue ${ }^{1}$ (WDS) lists more than 130,000 binaries which is the largest collection of binary stars. However, this catalogue shows that the key parameters for dynamical studies of planetary motion in binary star systems - i.e. the separation of the two stars and the eccentricity are known only for a small fraction of observed binary star systems. The lack of observational information causes difficulties for dynamical stability studies.

The detection of extra-solar planets in binary and multi-stellar systems showed that planetary companions are not restricted to single stars. A study by [4] using the Kepler data suggests an occurrence rate of coplanar circumbinary planets similar to that for single stars. The data of the observed extrasolar planets are collected in various catalogues. The most frequently used catalogues are the Extrasolar Planet Encyclopaedia ${ }^{2}$ [5], and the Exoplanet Orbit Database ${ }^{3}$ [6]. In addition, the Catalogue of Exoplanets in Binary Star Systems ${ }^{4}$ [7] lists only exoplanets in binary and multiple star systems which shows about 132 planets that have been detected in binary star systems so far. This number is certainly small compared to the nearly 3900 published exoplanets but we expect an increase of this number in the near future due to missions like GAIA, Plato, TESS CHEOPS, JWST, ELT and other promising observational projects.

In a binary star system one has to consider different types of planetary motion: P-type or circumbinary motion where the planet orbits both stars in a distant orbit; and S-type or circumstellar motion where the planet orbits one star. For the latter, the planetary orbit has to be close enough to the host-star to avoid perturbations from the secondary star. In both cases, the stellar parameters (i.e. mass, semi-major axis and eccentricity, inclination) play an important role for the formation, evolution and habitability of planets.

The formation of planets in the classical core-accretion scenario $[8,9]$ begins on the smallest scale, and lasts from sub-micron sized dust to Jupiter-like giant planets. Small planetesimals merge as a result of the coagulation of dust particles. Larger planetesimals and planetary embryos are formed by gravitationally assisted collisions of these smaller bodies. The end result of this process is the formation of terrestrial planets and solid cores of giant planets. On the other hand, there are a few still open issues of planet formation in the core accretion scenario, such as the existence of different barriers impeding the growth of dust particles, such as the meter size, bouncing and charge barriers $[10,11,12]$, and the timescale problem of giant planet formation, which is due to the combination of the fast type I migration of planetary cores [13] and the limited lifetime of protoplanetry disks [14]. These issues are also present in circumprimary disks, when the planet

\footnotetext{
${ }^{1}$ http://ad.usno.navy.mil/wds/

${ }^{2} \mathrm{http}: / /$ exoplanet.eu

${ }^{3} \mathrm{http} / / /$ exoplanets.org

${ }^{4}$ http://www.univie.ac.at/adg/schwarz/multiple.html
} 
forming gas disk is influenced by a stellar companion. Moreover, there are binary specific problems for planet formation:

(i) The truncation and distortion of the circumprimary disk by the stellar companion $[15,16]$. The main question in this context is certainly whether truncated disks contain enough solid and gaseous material to form gas giant and terrestrial planets. This issue has been numerically investigated by [17] and [18] who concluded for $\gamma$ Cephei-type systems that there is enough material in the truncated disk to form a gas giant.

(ii) Dust coagulation is problematic in mid-separation binary systems (see [11] because in the elliptically distorted protoplanetary disk the relative velocities between the colliding aggregates may be large enough to inhibit dust coagulation - instead of sticking together, the dust particles will destroy each other. This could be a real problem for the growth of planetesimals by mutual collisions in binary systems as it is well known that collisions of planetesimals are highly sensitive to their collision velocities $[19,20]$. The gravitational perturbations from the binary companion can excite the orbital eccentricities of the planetesimals, which will increase their impact velocities resulting in disruption instead of accretion [21,22].

[23] found that the combined effect of binary perturbations and local gas drag will result in the orbital alignment of similar sized planetesimal, which helps to reduce the collision velocities even for eccentric orbits. However, this mechanism cannot solve the planetesimal growth problem. [24] found that the alignment depends strongly on the size of planetesimals. Thus planetesimals of different sizes align their orbits to different orientations, consequently the mutual impact velocities between planetesimals of different sizes are too high and inhibit their growth [25].

To resolve this issue, namely that planet formation is indeed possible in mid-separation binaries, new possible routes of planetesimal growth should be found. A convenient solution would certainly be if planetesimals were born big, enough to resist the high velocity collisions. Such a mechanism would be favorable as it allows the direct formation of few hundred km-sized bodies from $\mathrm{cm}$-sized dust aggregates proposed by [26] when analyzing the current size frequency distribution of main belt asteroids in the Solar System. In disks around single stars a new concept has emerged, the particle trapping mechanism that can explain the formation of large planetesimals also overriding the infamous meter-size barrier. [27] proposed that particle concentrations in a turbulent disk may happen at small eddy scales, and may lead sometimes to the formation of selfgravitating sandpiles that will collapse to planetesimals between 1-100 km in size. A prerequisite of this mechanism is that dust aggregates should grow first to millimeter sizes. [28] showed that particle trapping and subsequent gravitational contraction of particle clouds can also happen at the scale of the largest turbulent eddies, leading to bodies between $100 \mathrm{~km}$ and $1000 \mathrm{~km}$ in size.

Accepting the fact that giant planet formation is possible even in $\gamma$ Cephei-type systems in the framework of the core accretion scenario, we may assume that isolated embryos can form as a result of an oligarchic growth of planetesimals, and during their final assembly, a system of terrestrial planets may form also in the binary case. Following this scenario, terrestrial planet formation has already been investigated by many authors as [29, 30, 31, 32] and [33] demonstrating that Earth-like planets could be formed in binary system within 10-100 Myr.

In this paper we discuss first the problems of accretion during the gas phase when the giant planets form. Then we show N-body simulations of terrestrial planet formation after the dissipation of the gas disk where the perturbations of a secondary star and a prior formed giant planet influence 
the planetary growth process.

\section{Planet formation during gas phase}

Understanding the various processes for planet formation in binary star systems is of high importance, since combined perturbations of an already developed gas giant planet and the secondary star can be quite effective in the case of rather tight binary systems, such as $\gamma$ Cephei [34, 35], GJ 86 [36, 37], or HD 41004 [38], in which massive exoplanets are known to orbit one of the stars. These discoveries have led to a growing interest in understanding planetary formation processes in general.

In such binary star systems, the secondary star together with the heavily truncated and possibly distorted disk influence the formation and evolution of planets throughout several stages of the planet-forming process. Disk truncation is caused by the companion star through gravitational interaction, as was shown by [39] and [40], where mainly the outer edge is influenced for circumstellar disks [15, 18]. The inner edge is affected in case of circumbinary disks [41]. To determine the border of stable motion in a binary star - which defines the truncation of the disk - one can use general stability studies like e.g. [42, 43, 44] The truncation shortens the lifetime of the disk and consequently limits the period in which gaseous planets can form. During the accretion of dust particles to km-sized planetesimals, the secondary star can force the shape and orientation of their orbits, which could stop planetary formation (see e.g. [45] and references therein). [46] summarized a number of observational surveys (e.g., IRAS and Spitzer satellites) that yield the disk lifetimes to be $\leq 10^{7}$ years, and the median disk dispersion time-scale is $2-3 \mathrm{Myr}$ as observed in various star forming regions.

\subsection{Fully interacting binary-star - disk -protoplanet system}

Recently, [47] performed a parameter study to analyze the interplay between a circumprimary gas disk, gravitationally interacting embryos, and a distant secondary star. For this investigation they developed a GPU-CPU 2D hydrodynamics grid code that combines hydrodynamic radiative disk computations with highly accurate $N$-body simulations. This code was used to probe the differences for planetary formation, when taking into account (i) binary-disk, (ii) binary-protoplanet, and (iii) the binary-protoplanet-disk interactions. By simulating a coplanar binary-disk system with a grid centered in the primary star, the interplay and evolution of the three components (stars, disk, and protoplanets) was investigated for $\gamma$ Cephei like configurations. For $\gamma$ Cephei, the primary's mass, $m_{A}$, and the secondary's mass, $m_{B}$, are $1.4 \mathrm{M}_{\odot}$ and $0.4 \mathrm{M}_{\odot}$, respectively, with a mutual distance of $\sim 20$ au and an eccentricity of 0.4 . The gas disk of $0.01 \mathrm{M}_{\odot}$ around the primary extended initially from 0.5 to 8 au with an initial density profile $\Sigma(r) \propto r^{-1}$, where $r$ denotes the distance from the primary. In addition, 2048 embryos with masses of $0.016 \mathrm{M}_{\odot}$ were distributed randomly around the primary. The arrangement of embryo-sized particles implicitly assumes that the planetesimal accretion phase was successful, which is far from being granted.

The investigation by [47] is of great importance for tight binary star systems, as it showed for the first time that close encounters between protoplanets and their interaction with frequently

\footnotetext{
${ }^{5}$ This simple density distribution places much material outside the Hill sphere of the primary star where it is strongly affected by the perturbations of the secondary.
} 
occurring spiral density waves in the gas disk induced by the secondary star lead to large scale momentum and energy exchange. These interactions might increase the semi-major axes and eccentricities of the protoplanets to high values and thus inhibit the agglomeration and merging of planetesimals and protoplanets. Moreover, changes in the mass-weighted disk eccentricity and the longitude of the disk pericenter cause variations in the dynamical evolution of the disk (see [18]) and might also affect the movement of protoplanets. This may either render planet formation impossible, or provide an environment where agglomeration is not strongly affected by the disk.

In this context, the study by [47] revealed the following information:

1. The evolution of the disk was not strongly affected by the particles when the total particleto-gas mass ratio was $10^{-2}$. However, a phase drift in the disk's mass-weighted eccentricity and argument of pericenter was observed between solutions where the protoplanets' influence is either incorporated or neglected. Moreover, the initially zero eccentricity of the disk increased to maximum values between 0.06 and 0.07 and reached 0.03 or 0.035 by the end of the computations (after 100 binary periods) in all the dynamical models they studied.

2. The evolution of interacting particles, perturbed by the stars and the gas disk, was studied for the first time with the combined hydrodynamical $-N$-body code. The eccentricities of the particles underwent strong variations, especially in the fully interacting model (up to $\sim 0.9$ in the first 1000 years). While in the model without a back-reaction from the disk, the evolution proceeded more slowly, and on average the eccentricity remained below 0.4. Moreover, the $N$-body relaxation processes occurred faster in the presence of a dynamically evolving and interacting gas disk, with average protoplanetary eccentricities almost twice as high as in non-interacting models. It was also shown that the relaxation time depends on the so-called smoothing parameter, which introduces a numerical viscosity and keeps eccentricities at low values. A quiet (low eccentricity) disk damps the particle eccentricities even when only disk gravity is taken into account.

3. The growth of particles yielded different collision probability distributions in the fully interacting model. Disruption dominated in this model in both time intervals that have been considered. ${ }^{6}$. Mergers were found to be more probable for the early time interval in the model that considered a quiet disk and the model without a back-reaction from the disk, though in the later time interval disruption dominated also in these dynamical models. Therefore, one can conclude that the growth from embryos to planets within a dynamically evolving gas disk is strongly altered by the dynamical evolution of the disk, which leads to a decreased probability for planet formation at least in the inner parts of the gas disk. This fact can be explained by the action of periodically occurring and inward-moving spiral waves in the disk that are exited by the secondary star together with a periodically varying disk eccentricity which leads to excitations of the embryos and thus higher encounter velocities.

There are many parameters that affect the outcome of the simulations, such as the gravitational smoothing parameter (which influences the disk-protoplanet and protoplanet-protoplanet interactions), the type of boundaries (reflecting, outflow, non-reflecting) of the grid in the hydrodynamic

\footnotetext{
${ }^{6}$ [47] compared two time intervals for merging and disruption: Interval $\mathrm{I}$ is from the 30th to the 40th binary orbit, and interval II is from the 50th to 60th binary orbit.
} 
part, different flux-limiter functions in the advection part of the code, and the orbit evolution of the secondary star. Therefore, future studies are needed to investigate their influences and shed more light on the problems of the early phase of planet formation in binary star systems.

However, we have observational evidence that giant planet formation is possible even in quite tight binaries. Consequently, a system of terrestrial planets may also form in binary star systems during the final assembly phase of planetesimals. It is a well established fact that giant planets play a decisive role for terrestrial planet formation [48].

\section{Planet formation after gas phase}

While gas planets have to form quite rapidly within the lifetime of the gas disk, terrestrial planet formation may take some tens to hundreds of millions of years. Numerical simulations by [32] showed terrestrial planet formation even in tight binary star systems. They performed numerical simulations for various binary configurations with separations between 20 and 40 au and compared their results with Solar System computations. From this study, they could conclude that terrestrial planets can easily form in such tight binary star systems where the final architecture of a planetary system depends on many parameters, e.g. the mass-ratio, the eccentricity and the semi-major axis of the binary. A comprehensive survey of the influence of the binary's dynamical and physical parameters on terrestrial planet formation is difficult, though. [32] have found a big diversity of planetary systems even with the restriction to tight binaries with stellar separations up to $40 \mathrm{au}$.

In our investigations we studied terrestrial planet formation in a wider range of binary configurations. We started the simulations from the end of the oligarchic growth, when the massive embryos already accreted the smaller planetesimals in their feeding zone. Our physical model is therefore the gravitational N-body problem, in which the only interaction between the involved bodies is the gravitational force.

\subsection{Initial set-up and Simulations}

To generate an initial swarm of embryo population we assume the usual power law profile for the surface density of solids $\Sigma_{\text {solid }}(r)=\Sigma_{0} \cdot r^{-\alpha}$, where $\Sigma_{0}=10 \mathrm{~g} / \mathrm{cm}^{2}$ is slightly larger than that of [49] for the Minimum Mass Solar Nebula (i.e. $\Sigma_{0}=7.1 \mathrm{~g} / \mathrm{cm}^{2}$ ). The power law index $\alpha=1$ fits the best to the protoplanetary disks' observations. The masses and the initial semi-major axes of the embryos have been calculated by using the concept of the isolation mass. An embryo is reaching the isolation mass by emptying its feeding zone, which is an annular region expressed in terms of its Hill radius. The total mass of planetesimals in this ring of width $\Delta a_{\max }=C r_{H i l l}$ is:

$$
m_{\text {iso }}=m_{\text {ring }}=2 \pi a C r_{\text {Hill }} \Sigma_{p}(a)
$$

where $C$ is a constant, and $\Sigma_{p}(a)$ is the surface density of the solid material in the ring at the distance $a$ from the star. And the Hill radius of a body with mass $m$ at distance $a$ from the host-star $M_{\star}$ is $r_{H i l l}=a\left(m / 3 M_{\star}\right)^{(1 / 3)}$. The distance of the closest embryo to the star is 0.3 au and $C=5$ which is slightly larger than the value $(2 \sqrt{3})$ suggested by [50]. 
The position of the $i$ th embryo with mass $m_{i}$ and distance $a_{i}$ is defined by the following iteration

$$
a_{i}=a_{i-1}+C a_{i-1}\left(\frac{m_{i-1}}{3 M_{\star}}\right)^{1 / 3}+C a_{i}\left(\frac{m_{i}}{3 M_{\star}}\right)^{1 / 3}
$$

for $a_{i} \leq a_{\max }$ with $a_{\max }=1.6$ or $2.6 \mathrm{au}$ (depending on the location of the giant planet which is either 2.5 or $5 \mathrm{au}$ ).

In all simulations we studied terrestrial planet formation around a Sun-like star and varied the mass of the perturbing secondary star between $0.4,0.7,1.0$ and $1.3 M_{\text {Sun }}$ (i.e. from $\mathrm{M}$ to $\mathrm{F}$ type stars). In most cases the perturbing star is less or equal massive than the host star except in case of the F type secondary star. In addition, the separation of the binary system $\left(a_{b i n}\right)$ is changed between 25 and $100 \mathrm{au}$, and the binary eccentricity $e_{b i n}$ is increased from 0 to 0.6 . As giant planet we used a Jupiter-mass planet $\left(m_{g p}=1 M_{\text {Jup }}\right)$ orbiting either at $a_{g p}=5$ au when $a_{b i n}>25$ au, or at $a_{g p}=2.5$ au for $a_{b i n}=25 \mathrm{AU}$. We reduced $a_{g p}$, in the latter case as the hosting protoplanetary disk is truncated heavily for such small binary separations, and it would be unlikely to form a giant planet at $5 \mathrm{au}$.

We integrate numerically the differential equations of the gravitational N-body problem in the barycentric coordinate system using a Bulirsch-Stoer (BS) integrator with adaptive stepsize control to be able to handle collisions between nearby bodies [51]. The condition for a collision of two bodies is: $d_{i, j}<\beta\left(r_{i}+r_{j}\right)$, where $d_{i, j}$ is the distance between bodies with radii $r_{i}$ and $r_{j}$, respectively, and $\beta$ is a factor of radius enhancement being a number of unity. We used $\beta=2.0$, which seems to be a good compromise, because according to our simulations this choice of $\beta$ accelerates numerical integration avoiding the very small time steps of the BS scheme needed to resolve collisions. On the other hand, the formation process has not been shortened significantly. The physical radii of the bodies have been calculated from the masses of the bodies using a bulk density of $\rho=2 \mathrm{~g} / \mathrm{cm}^{3}$. The mass and the initial velocity of the newly formed body are calculated assuming a perfectly inelastic collision using the center of mass approximation. In this way all collisions result in the merging of the bodies, no fragmentation and mass loss is incorporated in the model. We are aware of the fact that according to state-of-the-art collisional simulations using SPH (Smooth Particle Hydrodynamics) (see e.g. [52]), the outcome of collisions among planetary embryos could be a more complicated issue leading to destruction of these bodies. On the other hand, including the numerical treatment of fragmentation to our simulations would be very time consuming as this would increase the number of bodies in the N-body simulations.

\subsection{Evolution of the Embryo disk}

A typical behaviour of the embryos' semi-major axes when the formation process is not affected by a giant planet is shown in Figure 1. This simulation corresponds to the particular case when the separation and the eccentricity of the binary system are $a_{\text {bin }}=100$ au and $e_{\text {bin }}=0.01$. At the beginning of the simulation one can immediately observe the chaotic nature of the embryos' dynamics. Due to their mutual gravitational perturbations, the whole swarm of embryos expands to larger semi-major axes and their motion is strongly chaotic. In the example shown in Figure 1, first bodies are formed after roughly ten million years due to a series of consecutive collisions where these bodies accrete mass. The inner planets become seemingly stable after shorter time than the outer planets. On the other hand, a planet on an orbit with apparently constant semi-major axis 


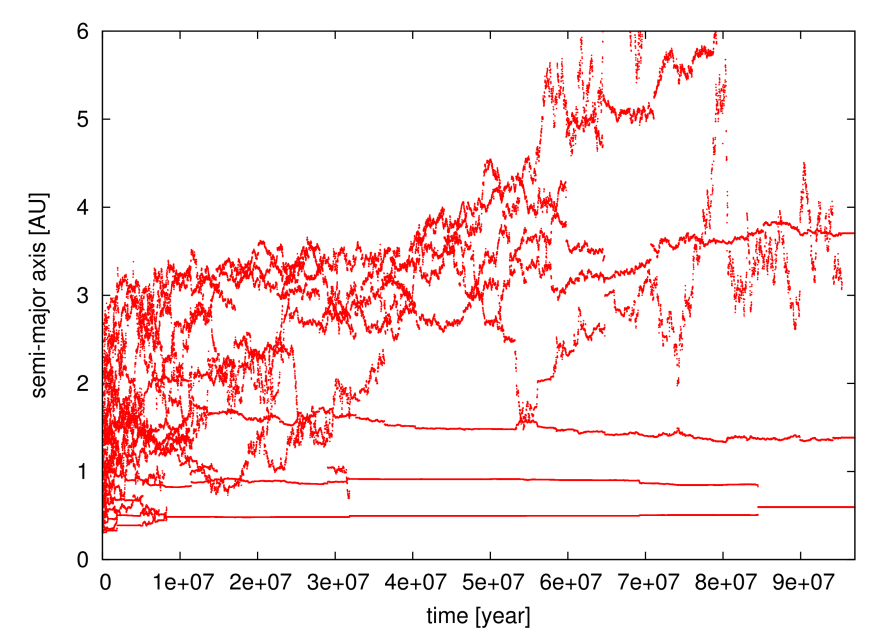

Figure 1: Evolution of the isolated embryo disk with initial positions between 0.3 and 2.6 au from the host-star in a binary star system with stellar separation $a_{b i n}=100$ au and binary eccentricity $e_{b i n}=0.01$, respectively. The evolutions of semi-major axes show that due to gravitational perturbations the embryos are pushed to larger semi-major axes and the whole population is expanding outwards.

can become chaotic due to pertubations leading to a collision with another already formed bigger body. The dynamics of the embryos in binary stars without giant planet seems to be only influenced when the binary separation is small, and the binary eccentricity and the stellar companion's mass are large. By increasing the stellar separation of the binary system the motion of the embryos will resemble more and more to the single star case.

\subsection{Perturbations of a giant planet}

From the dynamical point of view, it is a more interesting case when beside the stellar companion an already formed giant planet is also influencing the formation of terrestrial planets. We recall that our assumption on the embryo population is that the outward moving front of the oligarchic growth of embryos is at 2.6 AU. As one can see from Figure 1, the embryo population is expanding towards the giant planet, thus some members of this population will be affected by various mean motion resonances (MMRSs) with the giant planet leading to an apparent random walk through the different MMRs. As final outcome, many of the outer members of the swarm will be ejected from the system. This behaviour is illustrated in Figure 2 where the evolution of embryos is shown in a region perturbed by MMRs of the gas giant. The destabilization mechanism can be explained in terms of the different MMRs. It is known that the separatrices of an MMR form a 'V'-shape in the a - e plane (see [53] for more details) meaning that the region of the libration of the critical (resonant) angle is increasing with the eccentricity of the embryo being in MMR with the giant planet. For larger values of the eccentricity neighbouring resonances are overlapping each other resulting in a strongly chaotic behaviour for bodies being in this region of the phase space. Thus bodies with increased eccentricities moving in this region will be subject of strongly chaotic and unstable behaviour bringing them to close encounters with the giant planet. As a result, such bodies will be either ejected from the system or will leave this region. One can expect therefore that 
the ultimate result of terrestrial planet formation in binary star systems is strongly dependent on how the embryos' eccentricities are excited to higher values. The mechanisms responsible for the increase of eccentricities are the following: (i) gravitational self excitation of embryos, (ii) direct effects of the secondary stellar companion, (iii) indirect effects of the secondary star by increasing the giant planet's eccentricity, and finally (iv) secular resonances caused by the stellar companion and the giant planet in conjunction.

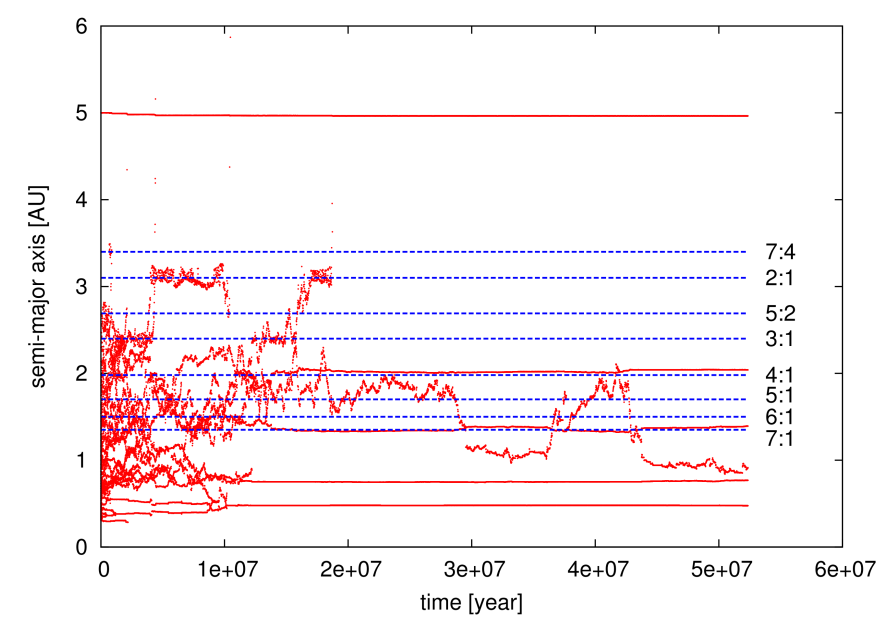

Figure 2: Same configuration as Figure 1 but with an already formed giant planet at 5 au (red horizontal line) which perturbs the planet forming region (indicated by the blue horizontal lines).

\subsection{The role of resonances}

In Figure 2 we show the perturbations of a binary configuration with stellar separation $a_{\text {bin }}=$ $100 \mathrm{au}$, binary eccentricity $e_{\text {bin }}=0.01$ and masses $M_{1}=1 M_{\text {Sun }}$, and $M_{2}=0.4 M_{\text {Sun }}$ which is among the studied systems that with the weakest perturbations from the secondary star. The behaviour of the embryos' semi-major axes is shown in Figure 2 which is mainly affected by the MMRs of the giant planet (indicated by the blue horizontal lines) and not by the combined effect of the secondary star and giant planet. Thus at least temporary resonant captures can happen into particular MMRs. During such a resonant capture the eccentricities of the involved bodies could be increased so that their orbit might end up in a region of the strong chaos. As a consequence, such bodies suffer close encounter with the giant planet and are ejected from the system. This behaviour is shown by two embryos in Figure 2 which are first captured into 3:1 MMR then they jump into 2:1 MMR and be ejected afterwards. It is noteworthy that in this system, there are two surviving bodies in the region filled densely by the different MMRs. One of these planets ends up in an orbit nearby the $4: 1$ resonance and the other planet is close to the $7: 1 \mathrm{MMR}$ which is in the so-called habitable zone $(H Z)$ of the primary star. Both planets are moving apparently in ordered orbits.

When we increase the eccentricity of this binary configuration to $e_{b i n}=0.6$ then, the secondary star approaches the primary star periodically to a distance of only $40 \mathrm{AU}$ which causes a periodic perturbation that increases the eccentricities of the giant planet and of the embryos. Even without a thorough study of the eccentricity excitation, one can immediately conclude from Figure 3 that 


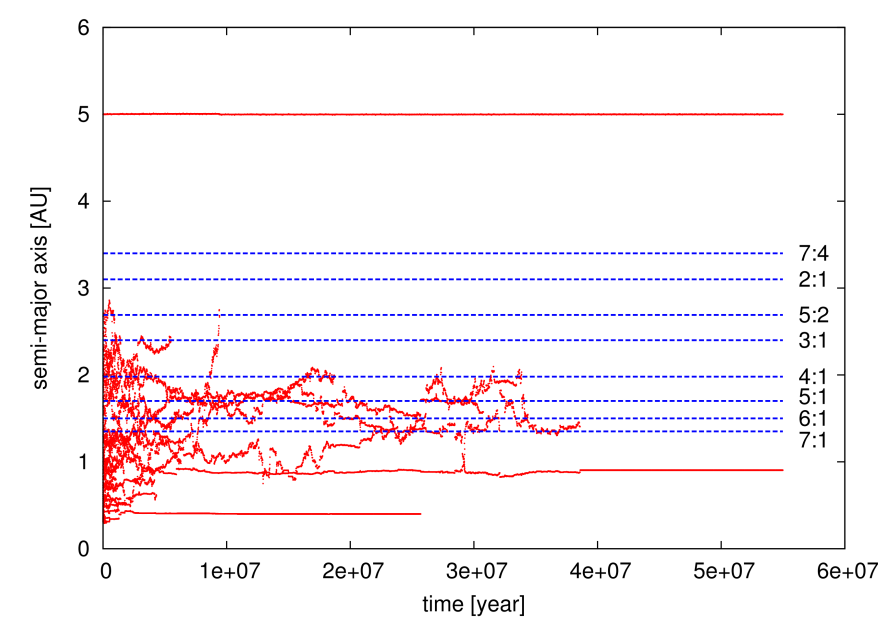

Figure 3: Same binary-star-planet configuration as in Figure 2 but with an increased binary eccentricity (0.6).

almost all embryos located beyond the $7: 1 \mathrm{MMR}$ are ejected from the planet forming region, and only one planet is formed at $\sim 0.9$ au which is close to the inner border of the HZ. Moreover, Figure 3 shows a sudden escape of the innermost body after about 26 Myrs which is due to a secular resonance (SR) where the eigen-frequencies of the embryo and of the giant planet are equal in this configuration. Recently, we have developed a Semi-Analytical Method for secular resonance detection - for details see $[54,55]$ which helps to locate and define secular resonances for circumstellar planetary motion in binary stars. In this case, a $1: 1$ SR with the giant planet's orbital precession frequency leads to an escape of this body due to the continually increase of the embryo's eccentricity.

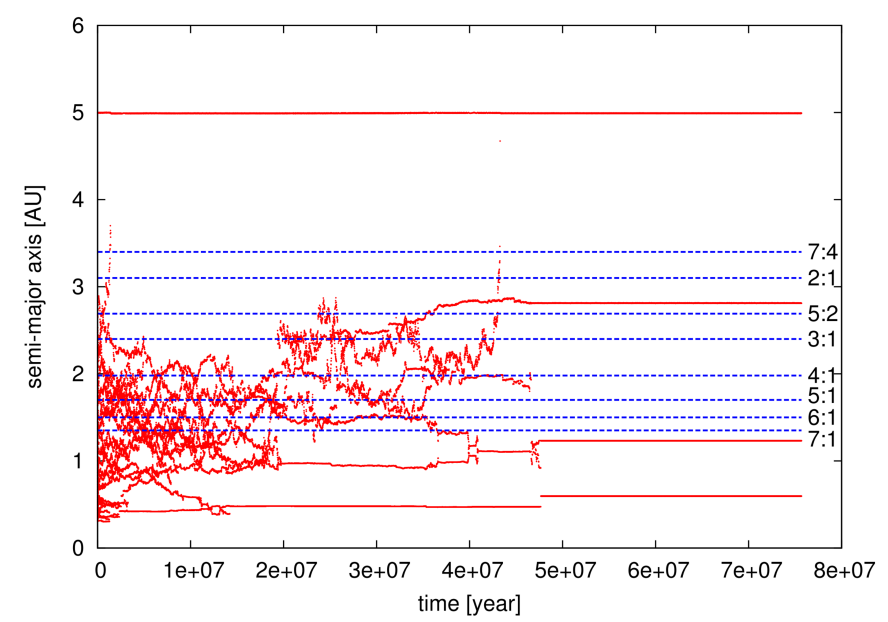

Figure 4: Same binary-star-planet configuration as in Figure 2 but with a smaller stellar separation of 50 au.

Instead of increasing the binary eccentricity one could decrease the stellar distance of the two stars to study stronger perturbations of the secondary star. Figure 4 shows the evolution of the 
embryos in the binary star - planet configuration with stellar separation $a_{b i n}=50$ au and $e_{b i n}=0.01$. Similarly to the case of the wider binary separation $\left(a_{b i n}=100 \mathrm{au}\right)$, the embryos are ejected from the system as a consequence of temporary captures to different resonances (see the ejections at $5: 2,3: 1$, and $4: 1$ MMRs). Finally, there are three surviving bodies: one in the resonant region close to the 5:2 MMR and two interior the $7: 1$ MMR. Comparing Figures 3 and 4 it seems that the increase of $e_{b i n}$ has a more severe effect on the dynamical behaviour of the embryos than the decrease of the binary separation.

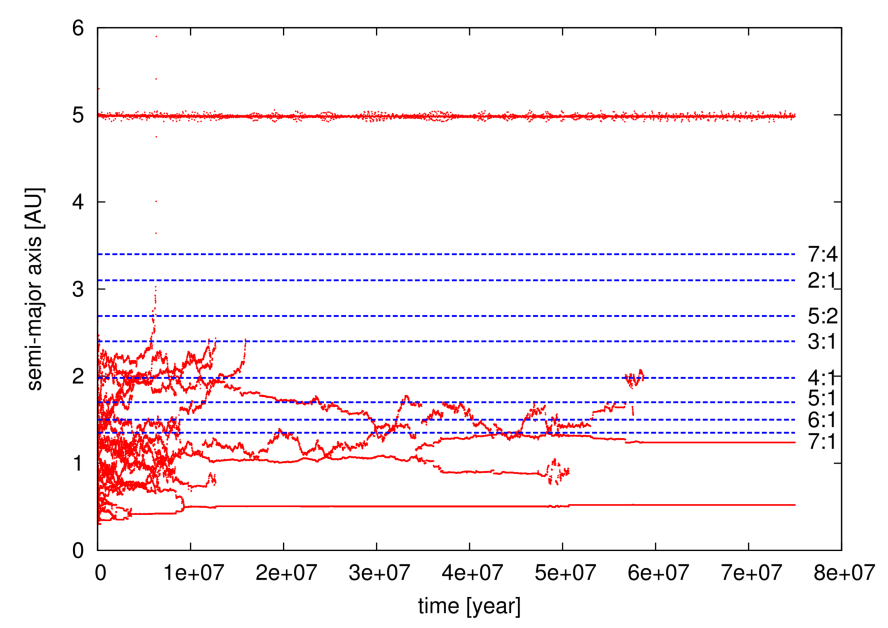

Figure 5: Same binary-star-planet configuration as in Figure 4 but with an increased binary eccentricity (0.6).

To investigate the effect of the binary's eccentricity further, we show the result for the binary configuration $a_{b i n}=50$ au for a higher eccentricity $e_{b i n}=0.6$ (see Figure 5). In that case, the secondary star approaches very close the primary (to $20 \mathrm{au}$ ), thus stronger periodic perturbations are visible for the giant planet and the embryos which result in a rapid destabilization of the outer embryo population (in which mainly the $4: 1$ and $3: 1$ MMRs are involved). Finally, only two planets survive, interior to the 7: $1 \mathrm{MMR}$. However, in this area we recognize also a sudden escape of a body after 50 Myrs which is caused by the $1: 1 \mathrm{SR}$ with the giant planet.

In order to verify how general the role of the MMRs is, we changed the semi-major axis of the giant planet to $a_{g p}=3.5$ au and performed simulations of the binary configuration for various stellar separations $\left(a_{b i n}=50,75\right.$ and $\left.100 \mathrm{au}\right)$ and eccentricities $e_{b i n}=0.01,0.2,0.4$ and 0.6. In all simulations we have found a similar behavior of the formed terrestrial planets as in the case $a_{g p}=5$ au. The terrestrial planets are formed in a region far from the strong MMRs and presumably from the strongly chaotic region. This assumption was confirmed by stability maps of the $(a, e)$-plane calculated with the aid of the chaos indicator RLI (i.e. Relative Lyapunov Indicator). The RLI was developed by Sándor and is known to be a fast tool to distinguish between regular and chaotic motion. For details we refer the reader to [56, 57]. From the results of our computations we can conclude that the presence of a giant planet causes a chaotic region due to the overlapping of various MMRs with the giant planet. This chaotic region plays a distinguished role in the outcome of terrestrial planet formation in binary stars. In all our simulations with a giant planet in the system where the stellar motion is nearly circular $\left(e_{b i n}=0.01\right)$ we observe the formation of a single planet 
in the area perturbed by MMRs, and mostly two planets interior to this region, where high order resonances (i.e. MMRs $>7: 1$ ) will not hamper the formation of a planet. In case of eccentric motion of the binary, generally no planet was formed in the MMR perturbed area and only one or two planets interior the $7: 1 \mathrm{MMR}$ can be observed.

\section{Final set-up of formed planetary systems}

However, in systems with larger stellar separations (e.g. $a_{b i n}=100 \mathrm{au}$ ) the chaotic region may contain areas of ordered motion where planets can form. This is due to the fact that the motion of the giant planet is less perturbed (i.e. its eccentricity remains small) by the secondary star which raise the possibility of unperturbed areas.
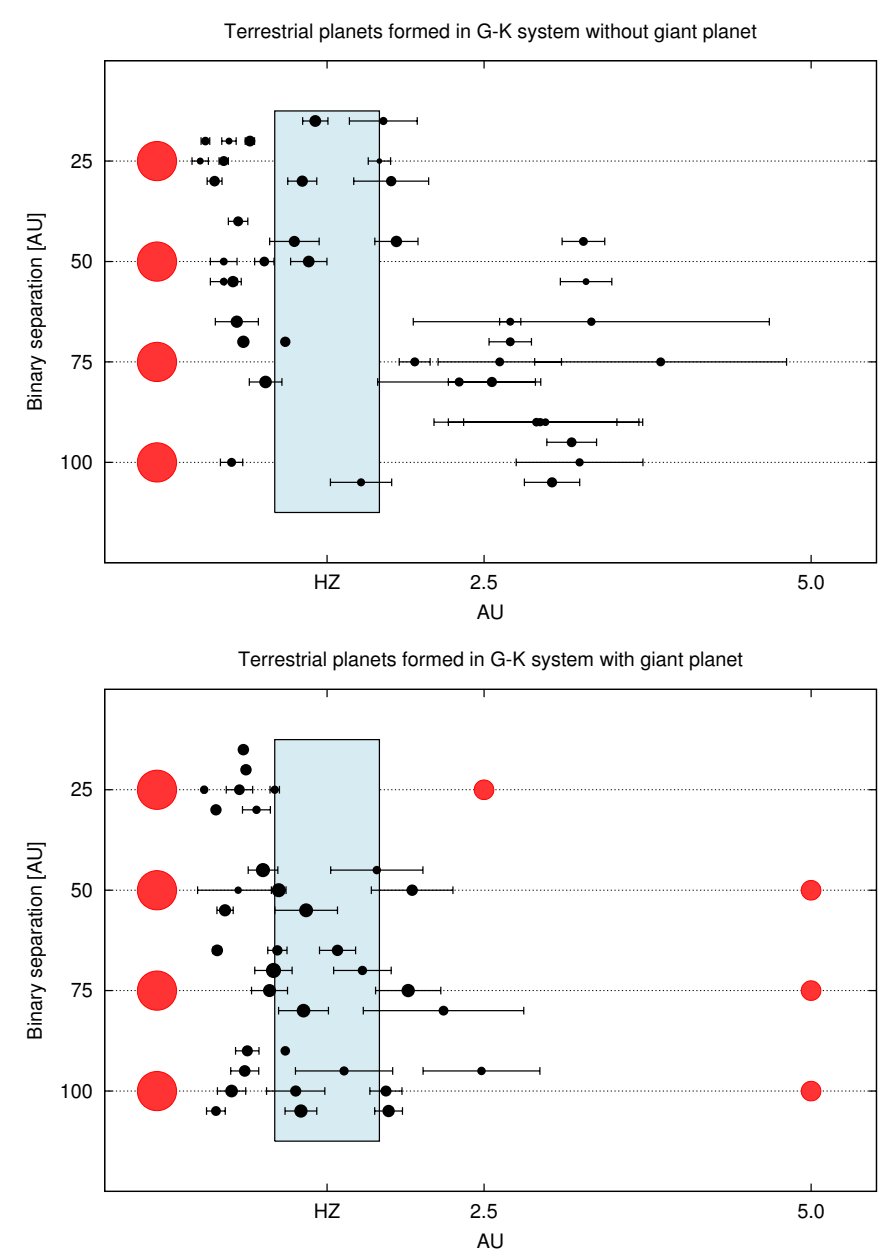

Figure 6: Terrestrial planets formed in binary star systems with different stellar separations (y-axis) and different binary eccentricities (i.e. different lines for a certain binary separation). The blue area marks the habitable zone (HZ) of the host-star (big red dot). Top panel shows the formed planets in systems without giant planet. Bottom panel shows the results of systems with giant planet (see the small red dot).

Figure 6 shows a comparison of simulations for a binary star system with stars of spectral types $\mathrm{G}$ and $\mathrm{K}$, with and without giant planet of one Jupiter-mass (marked by the smaller red circles in 
the lower panel). The horizontal axis symbolizes the distance to the host star, with the HZ being the light-blue area (i.e. between 0.95 and $1.67 \mathrm{au}$ ). The giant planet is located either at $2.5 \mathrm{au}$ (for tight binaries), or at about 5 au like in the Solar System.

Comparing the two figures, one can see that in binary systems without Jupiter (upper panel) the terrestrial planets formed after $100 \mathrm{Myr}$ indicate a higher diversity of planetary systems where even highly eccentric planetary motion is possible in the outer region. In the lower panel, the giant planet obviously limits the occurrence of terrestrial planets to the region within the 3:1 MMR at about $\sim 2.5 \mathrm{au}$. Another striking difference is that the orbits of formed planets are more ordered in the latter case, i.e. the orbits do not overlap. Both figures show the formation of terrestrial planets in the HZ for all stellar separations $a_{B}$. The point size indicates different masses of these planets, which depend on the frequency of collisions and, therefore, on the dynamical perturbations during the formation scenario.

Finally, we pay attention to the secondary star's mass which was varied between 0.4 and $1.3 M_{\text {Sun }}$. The results of these numerical simulations do not show strong differences in the dynamical evolution for the various masses. Thus we can conclude that an increase in eccentricity has the strongest influences on the dynamics of the protoplanetary disk while changes of the stellar separation and the secondary's mass are less effective.

\section{Conclusion}

Numerical simulations of the early stages of planetary formation in binary star systems figured out that we are faced with many open questions regarding where and how a planet can grow in such environments. There are many parameters that affect the outcome of the simulations, such as the smoothing parameter regarding the disk-protoplanet and protoplanet-protoplanet interaction, the type of boundary conditions (reflecting, outflow, non-reflecting) of the grid in the hydrodynamical part, different flux-limiter functions in the advection part of the code, and the orbital evolution of the secondary star. Therefore, future studies are needed to investigate their respective influence and shed more light on the problems of planet formation in binary star systems.

In contrast thereto, terrestrial planet formation using pure gravitational $N$-body simulations for embryo-sized bodies can be easily performed and result in one or several small planets depending on the initial binary-star-planet confirguration. If the binary star hosts also a giant planet, MMRs with respect to this planet play an important role as they restrict the region for the late stage formation and limits the eccentricity of the terrestrial planets to smaller values than in systems without giant planet.

In any case the initial configuration of a binary-star-planet system plays an important role since perturbations like mean motion and secular resonances will shape a system and trigger collisions of planetesimals and embryos and influence therefore the process of accretion and the final architecture of planetary systems.

\section{Acknowledgements}

The work of this publication is supported by the Austrian Science Fund (FWF). The authors acknowledge the support of the FWF NFN project S11608-N16 "Binary Star Systems and Habit- 
ability". M.G. was supported by the University of Vienna (FG 538003). E.P-L. would like to thank the organizers for the invitation to participate the APCS II Workshop.

\section{References}

[1] A. Duquennoy, M. Mayor and J.-L. Halbwachs, Multiplicity among solar type stars in the solar neighbourhood. I - CORAVEL radial velocity observations of 291 stars, A\&AS 88 (1991) 281.

[2] D. Raghavan, H. A. McAlister, T. J. Henry, D. W. Latham, G. W. Marcy, B. D. Mason et al., A Survey of Stellar Families: Multiplicity of Solar-type Stars, ApJS 190 (2010) 1 [1 007.0414$].$

[3] A. Tokovinin, From Binaries to Multiples. II. Hierarchical Multiplicity of F and G Dwarfs, AJ 147 (2014) 87 [1401.6827].

[4] D. J. Armstrong, H. P. Osborn, D. J. A. Brown, F. Faedi, Y. Gómez Maqueo Chew, D. V. Martin et al., On the abundance of circumbinary planets, MNRAS 444 (2014) 1873 [1404.5617].

[5] J. Schneider, C. Dedieu, P. Le Sidaner, R. Savalle and I. Zolotukhin, Defining and cataloging exoplanets: the exoplanet.eu database, A\&A 532 (2011) A79 [1106.0586].

[6] E. Han, S. X. Wang, J. T. Wright, Y. K. Feng, M. Zhao, O. Fakhouri et al., Exoplanet Orbit Database. II. Updates to Exoplanets.org, PASP 126 (2014) 827 [1409. 7709 ].

[7] R. Schwarz, B. Funk, R. Zechner and Á. Bazsó, New prospects for observing and cataloguing exoplanets in well-detached binaries, MNRAS 460 (2016) 3598 [1608 . 00764 ].

[8] P. Bodenheimer and J. B. Pollack, Calculations of the accretion and evolution of giant planets The effects of solid cores, 67 (1986) 391.

[9] J. B. Pollack, O. Hubickyj, P. Bodenheimer, J. J. Lissauer, M. Podolak and Y. Greenzweig, Formation of the Giant Planets by Concurrent Accretion of Solids and Gas, 124 (1996) 62.

[10] J. Blum and G. Wurm, The Growth Mechanisms of Macroscopic Bodies in Protoplanetary Disks, 46 (2008) 21.

[11] A. Zsom, C. W. Ormel, C. Güttler, J. Blum and C. P. Dullemond, The outcome of protoplanetary dust growth: pebbles, boulders, or planetesimals? II. Introducing the bouncing barrier, 513 (2010) A57 [1001.0488].

[12] S. Okuzumi, Electric Charging of Dust Aggregates and its Effect on Dust Coagulation in Protoplanetary Disks, 698 (2009) 1122 [0901.2886].

[13] W. R. Ward, Protoplanet Migration by Nebula Tides, 126 (1997) 261.

[14] K. E. Haisch, Jr., E. A. Lada and C. J. Lada, Disk Frequencies and Lifetimes in Young Clusters, 553 (2001) L153 [astro-ph/0104347].

[15] W. Kley and R. P. Nelson, Early Evolution of Planets in Binaries: Planet-Disk Interaction, in Planets in Binary Star Systems (N. Haghighipour, ed.), (Dordrecht), pp. 135-164, Springer Netherlands, 2010, DOI.

[16] Z. Regály, Z. Sándor, C. P. Dullemond and L. L. Kiss, Spectral signatures of disk eccentricity in young binary systems. I. Circumprimary case, 528 (2011) A93 [1101.1820].

[17] H. Jang-Condell, M. Mugrauer and T. Schmidt, Disk Truncation and Planet Formation in $\gamma$ Cephei, 683 (2008) L191 [0 807.4472 ]. 
[18] T. W. A. Müller and W. Kley, Circumstellar disks in binary star systems. Models for $\gamma$ Cephei and $\alpha$ Centauri, A\&A 539 (2012) A18 [1112.1845].

[19] W. Benz and E. Asphaug, Catastrophic Disruptions Revisited, Icarus 142 (1999) 5 [astro-ph/9907117].

[20] S. T. Stewart and Z. M. Leinhardt, Velocity-Dependent Catastrophic Disruption Criteria for Planetesimals, ApJL 691 (2009) L133.

[21] T. A. Heppenheimer, On the formation of planets in binary star systems, A\&A 65 (1978) 421.

[22] D. P. Whitmire, J. J. Matese, L. Criswell and S. Mikkola, Habitable Planet Formation in Binary Star Systems, Icarus 132 (1998) 196.

[23] F. Marzari and H. Scholl, Planetesimal Accretion in Binary Star Systems, ApJ 543 (2000) 328.

[24] P. Thébault, F. Marzari and H. Scholl, Relative velocities among accreting planetesimals in binary systems: The circumprimary case, Icarus 183 (2006) 193 [arXiv: astro-ph/ 0602046 ].

[25] P. Thébault, F. Marzari and H. Scholl, Planet formation in $\alpha$ Centauri A revisited: not so accretion friendly after all, 388 (2008) 1528 [0806.0819].

[26] A. Morbidelli, W. F. Bottke, D. Nesvorný and H. F. Levison, Asteroids were born big, 204 (2009) 558 [0907.2512].

[27] J. N. Cuzzi, R. C. Hogan and K. Shariff, Toward Planetesimals: Dense Chondrule Clumps in the Protoplanetary Nebula, 687 (2008) 1432 [0 804.3526$].$

[28] A. Johansen, J. S. Oishi, M.-M. Mac Low, H. Klahr, T. Henning and A. Youdin, Rapid planetesimal formation in turbulent circumstellar disks, 448 (2007) 1022 [0708.3890].

[29] M. Barbieri, F. Marzari and H. Scholl, Formation of terrestrial planets in close binary systems: The case of alpha Centauri A, A\&A 396 (2002) 219 [a stro-ph/ 0209118 ].

[30] E. V. Quintana and J. J. Lissauer, Terrestrial planet formation surrounding close binary stars, 185 (2006) 1 [astro-ph/0607222].

[31] E. V. Quintana, F. C. Adams, J. J. Lissauer and J. E. Chambers, Terrestrial Planet Formation around Individual Stars within Binary Star Systems, ApJ 660 (2007) 807 [astro-ph / 0701266 ].

[32] N. Haghighipour and S. N. Raymond, Habitable Planet Formation in Binary Planetary Systems, ApJ 666 (2007) 436 [astro-ph/ 0702706 ].

[33] J. M. Guedes, E. J. Rivera, E. Davis, G. Laughlin, E. V. Quintana and D. A. Fischer, Formation and Detectability of Terrestrial Planets around $\alpha$ Centauri B, ApJ 679 (2008) 1582 [0 802 . 3482 ].

[34] A. P. Hatzes, W. D. Cochran, M. Endl, B. McArthur, D. B. Paulson, G. A. H. Walker et al., A Planetary Companion to $\gamma$ Cephei A, ApJ 599 (2003) 1383 [arXiv: astro-ph/0305110].

[35] R. Neuhäuser, M. Mugrauer, M. Fukagawa, G. Torres and T. Schmidt, Direct detection of exoplanet host star companion $\gamma$ Cep B and revised masses for both stars and the sub-stellar object, A\&A 462 (2007) 777 [arXiv:astro-ph/0611427].

[36] N. C. Santos, M. Mayor, D. Naef, F. Pepe, D. Queloz, S. Udry et al., The CORALIE survey for Southern extra-solar planets. IV. Intrinsic stellar limitations to planet searches with radial-velocity techniques, A\&A 361 (2000) 265.

[37] A.-M. Lagrange, H. Beust, S. Udry, G. Chauvin and M. Mayor, New constrains on Gliese 86 B. VLT near infrared coronographic imaging survey of planetary hosts, A\&A 459 (2006) 955. 
[38] S. Zucker, T. Mazeh, N. C. Santos, S. Udry and M. Mayor, Multi-order TODCOR: Application to observations taken with the CORALIE echelle spectrograph. II. A planet in the system HD 41004, A\&A 426 (2004) 695.

[39] P. Artymowicz and S. H. Lubow, Dynamics of binary-disk interaction. 1: Resonances and disk gap sizes, ApJ 421 (1994) 651.

[40] G. J. Savonije, J. C. B. Papaloizou and D. N. C. Lin, On Tidally Induced Shocks in Accretion Discs in Close Binary Systems, MNRAS 268 (1994) 13.

[41] R. R. Rafikov, Building Tatooine: Suppression of the Direct Secular Excitation in Kepler Circumbinary Planet Formation, ApJL 764 (2013) L16 [1212 . 2217].

[42] M. J. Holman and P. A. Wiegert, Long-Term Stability of Planets in Binary Systems, AJ 117 (1999) 621 [astro-ph/9809315].

[43] E. Pilat-Lohinger and R. Dvorak, Stability of S-type Orbits in Binaries, Celestial Mechanics and Dynamical Astronomy 82 (2002) 143.

[44] E. Pilat-Lohinger, B. Funk and R. Dvorak, Stability limits in double stars. A study of inclined planetary orbits, A\&A 400 (2003) 1085.

[45] P. Thebault, Against all odds? Forming the planet of the HD 196885 binary, CMDA 111 (2011) 29 [1103.3900].

[46] J. P. Williams and L. A. Cieza, Protoplanetary Disks and Their Evolution, Annu. Rev. Astron. Astrophys. 49 (2011) 67 [1103.0556].

[47] M. Gyergyovits, S. Eggl, E. Pilat-Lohinger and C. Theis, Disc-protoplanet interaction. Influence of circumprimary radiative discs on self-gravitating protoplanetary bodies in binary star systems, A\&A 566 (2014) A114 [1405.5056].

[48] H. F. Levison and C. Agnor, The Role of Giant Planets in Terrestrial Planet Formation, AJ 125 (2003) 2692.

[49] C. Hayashi, Structure of the Solar Nebula, Growth and Decay of Magnetic Fields and Effects of Magnetic and Turbulent Viscosities on the Nebula, Progress of Theoretical Physics Supplement 70 (1981) 35 .

[50] J. J. Lissauer, Planet formation, 31 (1993) 129.

[51] Z. Sándor, W. Lyra and C. P. Dullemond, Formation of Planetary Cores at Type I Migration Traps, 728 (2011) L9 [1101.0942].

[52] T. I. Maindl, R. Dvorak, C. Schäfer and R. Speith, Fragmentation of colliding planetesimals with water content, in Complex Planetary Systems, Proceedings of the International Astronomical Union, vol. 310 of IAU Symposium, pp. 138-141, July, 2014, 1409.2298 , DOI.

[53] C. D. Murray and S. F. Dermott, Solar system dynamics. Cambridge University Press, 1999.

[54] E. Pilat-Lohinger, Á. Bazsó and B. Funk, A Quick Method to Identify Secular Resonances in Multi-planet Systems with a Binary Companion, AJ 152 (2016) 139.

[55] Á. Bazsó, E. Pilat-Lohinger, S. Eggl, B. Funk, D. Bancelin and G. Rau, Dynamics and habitability in circumstellar planetary systems of known binary stars, MNRAS 466 (2017) 1555 [1605. 06769 ].

[56] Z. Sándor, B. Érdi, A. Széll and B. Funk, The Relative Lyapunov Indicator: An Efficient Method of Chaos Detection, Celestial Mechanics and Dynamical Astronomy 90 (2004) 127.

[57] Z. Sándor, Á. Süli, B. Érdi, E. Pilat-Lohinger and R. Dvorak, A stability catalogue of the habitable zones in extrasolar planetary systems, 375 (2007) 1495. 